\title{
Pesquisa colaborativa em design para aproveitamento de resíduos de açaí em comunidades locais: delimitação do estado da arte. ${ }^{1}$
}

\author{
Investigación colaborativa en diseño para el uso de residuos de açaí en \\ comunidades locales: delimitando el estado del arte
}

\author{
Collaborative research in design for the use of açaí residues in local \\ communities: delimiting the state of the art
}

\author{
Caroline Pedraça Santos ${ }^{2}$ \\ Raquel Gomes Noronha ${ }^{3}$
}

\begin{abstract}
Resumo
O Brasil produz mais de 221 mil toneladas de açaí por ano, das quais 161 mil toneladas correspondem aos resíduos oriundos do seu despolpamento. Estudos relacionados ao aproveitamento do caroço e da fibra do açaí indicam o uso dos resíduos no setor agrícola, na fabricação de produtos medicinais e na engenharia de materiais. No entanto, as pesquisas sobre esses processos não incluem os conhecimentos tradicionais das comunidades produtoras de açaí no desenvolvimento de soluções para o aproveitamento desses resíduos. Nota-se que os valores culturais pertencentes a essas comunidades são ignorados na construção da educação acadêmica quando se trata da busca por inovações no campo da pesquisa. Neste sentido, foi realizada uma Revisão Sistemática de Literatura (RSL) para identificação de pesquisas baseadas em práticas colaborativas envolvendo a corpo acadêmico e a comunidade local. Obtendo como resultado dois artigos que apontam para a importância do conhecimento tácito no desenvolvimento de pesquisas que tenham como objetivo o manejo de recursos naturais, além de abordarem a necessidade de proteção de conhecimentos tradicionais. Apesar dos estudos apontarem para novos caminhos nas pesquisas acadêmicas, verifica-se a existência de uma lacuna no que se refere ao desenvolvimento de materiais no campo do design a partir de práticas colaborativas envolvendo pesquisadores e comunidades tradicionais. Tais práticas podem abrir novos caminhos para a construção da educação e da pesquisa científica e ainda fortalecer a cultura tradicional de comunidades locais.
\end{abstract}

Palavras-Chave: valorização da cultura local; práticas colaborativas; resíduos do açaí.

\section{Resumen}

Brasil produce más de 221 mil toneladas de açaí por año, de las cuales 161 mil toneladas corresponden a los residuos de su pulpa. Los estudios relacionados con el uso de semillas y fibra de açaí indican el uso de residuos en el sector agrícola, en la fabricación de medicamentos y en la ingeniería de materiales. Sin embargo, la investigación sobre estos procesos no incluye el conocimiento tradicional de las comunidades productoras de açaí en el desarrollo de soluciones para el uso de estos residuos. Se observa que los valores culturales que pertenecen a estas comunidades se ignoran en la construcción de la educación académica cuando se trata de buscar innovaciones en el campo de la investigación. En este sentido, se llevó a cabo una Revisión sistemática de literatura (RSL) para identificar investigaciones basadas en prácticas de colaboración que involucran al cuerpo

\footnotetext{
1 Artigo apresentado no II Congresso Internacional Online de Estudos sobre Culturas, na modalidade online, 2020 .

2 Mestranda em Design; Universidade Federal do Maranhão - UFMA; São Luís, Maranhão, Brasil; carolpedraca@gmail.com

${ }^{3}$ Doutora em Ciências Sociais; Universidade do Estado do Rio de Janeiro - UERJ; Rio de Janeiro, Rio de Janeiro, Brasil; docente permanente do Programa de Pós Graduação em Design da Universidade Federal do Maranhão - UFMA. raquel.noronha@ufma.br
} 
académico y la comunidad local. Como resultado, dos artículos señalan la importancia del conocimiento tácito en el desarrollo de la investigación dirigida al manejo de los recursos naturales, además de abordar la necesidad de proteger el conocimiento tradicional. A pesar de los estudios que apuntan a nuevos caminos en la investigación académica, existe una brecha con respecto al desarrollo de materiales en el campo del diseño basado en prácticas de colaboración que involucran a investigadores y comunidades tradicionales. Dichas prácticas pueden abrir nuevos caminos para la construcción de la educación y la investigación científica y también fortalecer la cultura tradicional de las comunidades locales.

Palabras claves: valorización de la cultura local; prácticas colaborativas; residuos de açaí.

\begin{abstract}
Brazilian country produces more than 221 thousand tons of açaí per year, these 161 thousand tons correspond to the residues from its pulp. Studies related to the use of açaí's seed and fiber, indicate it's use as residues in the agricultural sector, in the manufacture of medicinal products and in the engineering of materials. However, research on these processes does not include the traditional knowledge of the açaí's producing communities in the development of solutions for the use of these residues. It's observed that the cultural values belonging to these communities are ignored in the construction of academic education when it comes to the demand for innovations in the research's field. In this sense, a Systematic Literature Review (RSL) was carried out to identify research based on collaborative practices involving the academic body and the local community. As a result, two articles point to the importance of tacit knowledge in the development of research aimed at the management of natural resources, in addition to addressing the need to protect traditional knowledge. Despite the studies pointing to new paths in academic research, there is a gap regarding the development of materials in the field of design based on collaborative practices involving researchers and traditional communities. These practices can open new paths for the construction of education and scientific research and also strengthen the traditional culture of local communities.
\end{abstract}

Keywords: valorization of local culture; collaborative practices; açaí waste.

\title{
1. Introdução
}

As nomenclaturas utilizadas para designar o fruto do açaí são bem diversas, variando de um local para o outro. Em Belém do Pará, o fruto é amplamente conhecido como açaí e no Amazonas como açaí-de-planta (OLIVEIRA; FARIAS NETO, 2019). Já no Maracanã, bairro de fortes tradições culturais e onde a produção do fruto se destaca na cidade de São Luís, Maranhão, é conhecido como juçara. Por esse motivo a nomenclatura a ser adotada neste artigo seguirá o que prevalece na literatura científica, sendo o açaizeiro (Euterpe Oleracea) predominante em toda região amazônica, norte e nordeste do país e a palmeira da juçara (Euterpe Edulis) nativa da Mata Atlântica, região sul e sudeste do Brasil (MARÇAL et al., 2015, p. 693).

Existe uma importante diferenciação entre as duas palmeiras, o seu consumo alimentar, pois a palmeira da juçara (Euterpe Edulis) é tradicionalmente utilizada para a extração do palmito e por isso está na lista das espécies ameaçadas de extinção, tendo em vista que o material é retirado do caule da árvore. Marçal et al. (2015, p. 693) explicam que algumas iniciativas têm sido realizadas a fim de promoverem o cultivo da juçara para o consumo de seu fruto, da mesma forma como já é feito com o açaí que se destaca como a 
parte mais importante economicamente da espécie (OLIVEIRA et al., 2002, p. 1). As pesquisas indicam que ambos os frutos apresentam em suas polpas, elevados teores antioxidantes conferindo às mesmas a classificação de "superfruta" (SANTOS et al. apud MARÇAL et al., 2015, p. 693). A essa característica se atribui o sucesso do consumo da polpa do açaí em todo Brasil e também fora dele.

O açaí extraído no Brasil, especialmente nas regiões norte e nordeste, tem sido exportado para diversos países, disseminando esta importante tradição alimentar em outros lugares do mundo. Oliveira et al. (2002, p. 14) afirma que amostras da polpa e de seus derivados têm sido enviadas especialmente para a Áustria, Alemanha, Estados Unidos, Japão e países da Europa. Os dados do Instituto Brasileiro de Geografia e Estatística (IBGE) apresentados na tabela 1, abaixo, demonstram a quantidade produzida por região:

Tabela 1: Quantidade produzida na extração vegetal (Toneladas)/Região

\begin{tabular}{ll}
\hline Brasil e Grande Região & Açaí (Fruto) \\
\hline Brasil & 221.646 \\
\hline Norte & 204.011 \\
\hline Nordeste & 17.635 \\
\hline
\end{tabular}

Fonte: adaptado de IBGE (2018, não paginado)

Por outro lado, a grande questão que envolve o presente artigo relaciona-se com os resíduos oriundos do processo de extração da polpa do açaí. De acordo com Oliveira et al. (2002, p. 4), o caroço e a fibra resultantes da extração da polpa correspondem ao endocarpo, representando $73,46 \%$ do peso da fruta. Considerando que a produção anual no Brasil ultrapassa as 221 mil toneladas, tem-se o total de 161 mil toneladas de resíduos. Neste contexto, o estado do Maranhão apresenta-se como o terceiro produtor de açaí do Brasil, com uma produção de mais de 12 mil toneladas correspondentes aos resíduos do fruto.

Nesse cenário, existe uma tendência mundial na busca por compósitos que utilizem as fibras naturais na indústria de materiais, construção civil, automobilística e aeronáutica que apresentam baixo custo, baixa toxidade e baixo consumo energético. A utilização das fibras naturais em relação às sintéticas apresenta aspectos ecológicos e sociais positivos. De acordo com Martins et al. $(2009$, p. 1151,1156$)$ tanto o caroço quanto a fibra do açaí resistem a temperaturas superiores a $150^{\circ}$, níveis de aquecimento alcançado na moldagem de material polimérico, ou seja, podem ser utilizados na indústria de embalagem. Assim como Mesquita et al. (2018, p. 650) verificaram a viabilidade da sua utilização na fabricação de painéis 
aglomerados. Tais pesquisas indicam que é possível utilizar industrialmente os resíduos de açaí.

No entanto, como poderá ser visto na pesquisa em epígrafe, apesar de todos os testes feitos em laboratório, não existem estudos que envolvam a própria comunidade extratora de açaí na produção de novos materiais, em processos que buscam o beneficiamento desses resíduos. Considerando que, em grande parte, a exploração do fruto do açaí é realizada pela própria comunidade constituída de pequenos produtores, mostra-se urgente a necessidade de desenvolver pesquisas que envolvam essas pessoas no processo de produção de materiais a partir dos resíduos do açaí.

Neste contexto, métodos controlados com a presença de processos altamente complexos provavelmente não se adequariam à realidade local. Sendo assim, para que um processo possa fazer sentido para uma determinada comunidade, deve ser desenvolvido em colaboração com a mesma. Sobre esse aspecto, Manzini (2017, p.49) explica que além da resolução de problema, o design deve ser compreendido a partir do campo da cultura, da linguagem e do significado. Para tanto, somente através de uma experiência, o design pode gerar sentido. De acordo com Sanders (2002, não paginado) "as pessoas querem se expressar e participar direta e proativamente no desenvolvimento do design" (tradução nossa). Ou seja, as opiniões e percepções já não devem mais ser coletadas ao final de um processo, mas devem fazer parte dele. A autora explica que diante de um cenário de relações cada vez mais horizontalizadas, as pessoas podem expressar o que e como querem. O que reforça ainda mais a importância da participação da comunidade no processo de desenvolvimento de novos compósitos, tendo em vista que o principal objetivo é possibilitar que a mesma se aproprie do processo produtivo.

Considerando a importância de se desenvolver um novo material em colaboração com a comunidade, faz-se necessário que além das propriedades dos materiais, sejam conhecidas também suas qualidades, as quais devem ser consideradas a partir da percepção comunidade. Ingold (2015, p. 66) explica que para além das propriedades que podem ser mensuráveis através de testes em laboratório, as qualidades vêm "da experiência de uma vida de trabalho com o material. Esse é um conhecimento nascido da percepção sensorial e do engajamento prático" (INGOLD, 2015, p. 66). Neste sentido, o conhecimento tácito da comunidade sobre determinado alimento ou material, pode ser extremamente enriquecedor para a pesquisa acadêmica. Logo, não só a comunidade pode se beneficiar com os resultados da pesquisa, mas toda a sociedade. 
Buscamos neste artigo compreender se é possível a utilização do caroço e da fibra, resultantes da extração da polpa do açaí, na produção de novos materiais a partir de intervenções de design realizadas em colaboração com a comunidade do Maracanã. O bairro é rico em intensas tradições culturais relacionadas ao bumba meu boi e a outras celebrações populares como a tradicional festa da juçara (açaí) relacionada ao costume de festejar a safra que ocorre no mês de outubro (REPORTER..., 2019).

Para se chegar a este objetivo foi realizada uma Revisão Sistemática de Literatura RSL para delinear o estado da arte sobre os métodos para o aproveitamento de resíduos do açaí na produção de novos compósitos. Para isso foram selecionadas duas bases de dados por um determinado período de tempo, na base de dados da CAPES do ano de 2009 a 2019 e na base de dados WEB OF SCIENCE do ano de 2014 a 2019. Para a pesquisa, considerou-se os resíduos gerados após o despolpamento do fruto e suas possíveis utilizações tendo em vista a proposição de novos materiais através de práticas colaborativas no campo do design. Dessa maneira, buscando a valorização da cultura tangíveis (resíduos) e intangível (conhecimento tácito) da comunidade envolvida em um processo colaborativo como nos propõe Spinuzzi (2005, p. 164).

\section{Metodologia}

Segundo Galvão e Pereira (2014, p. 183) a importância da Revisão Sistemática de Literatura (RSL) está em oferecer um caminho coerente para esclarecer controvérsias, tomando como base apenas os estudos de melhor qualidade sobre um determinado assunto. $\mathrm{O}$ processo tem início com a identificação do problema, seguindo para a elaboração de um protocolo de pesquisa, o qual deve apresentar os critérios utilizados para a realização da RSL. Todo o processo deve ser empreendido com rigor metodológico e seguir um fluxo prédeterminado de etapas (SANCHES et al, 2018, p. 135), as quais se dão da seguinte maneira: localizando os estudos, selecionando os mais relevantes, avaliando a qualidade das pesquisas, compilando dados individuais, resumindo os resultados e, por fim, gerando um relatório sobre a revisão desenvolvida (JBIEBNM, 2004, p. 1).

O protocolo da RSL é desenvolvido a partir da questão de pesquisa formulada após a delimitação do problema (JBIEBNM, 2004, p. 2). Dentre outras informações, o protocolo deve conter as palavras-chave que serão inseridas nas bases de dados para identificação de pesquisas, as quais serão analisadas com base em critérios de inclusão e exclusão. Esses critérios devem ser explícitos para que possam ser replicáveis por outros pesquisadores em 
caso de repetição do processo utilizado (GALVÃO; PEREIRA, 2014, p. 183).

Sendo assim, temos a seguinte questão de pesquisa: quais metodologias estão sendo aplicadas nas pesquisas que possuem como objetivo gerar novos compósitos a partir dos resíduos do açaí? Como desenvolver novas pesquisas que incluam a comunidade através de processos que envolvam as práticas colaborativas?

Com o objetivo de localizar estudos que possam responder a estas perguntas, um protocolo de pesquisa foi gerado a partir dos parâmetros citados no quadro 1:

\begin{tabular}{|c|c|c|}
\hline & Base de dados CAPES & $\begin{array}{l}\text { Busca avançada } \\
\text { Índice boleano: AND } \\
\text { Data de publicação: últimos } 10 \text { anos } \\
\text { Tipo de material: todos os itens } \\
\text { Língua: qualquer idioma }\end{array}$ \\
\hline b. & Base de dados WEB OF SCIENCE & $\begin{array}{l}\text { Pesquisa básica - por tópico } \\
\text { Índice boleano: AND } \\
\text { Tempo estipulado: últimos } 5 \text { anos } \\
\text { Língua: inglês }\end{array}$ \\
\hline c. & Critérios para análise do material & $\begin{array}{l}\text { Ordem: título, resumo e palavras chaves. } \\
\text { Quando em um desses tópicos era identificada informação relevante, uma } \\
\text { terceira busca era realizada. De forma pontual era realizada uma busca em } \\
\text { todo texto pelas palavras-chave através da utilização da ferramenta de busca } \\
\text { [“control" + "F”]. Caso fossem encontradas informações sobre as } \\
\text { propriedades do caroço, da casca ou sobre a utilização desses resíduos no } \\
\text { campo do design, o artigo era selecionado. }\end{array}$ \\
\hline d. & Critérios de inclusão & $\begin{array}{l}\text { Pesquisas que abordem o aproveitamento dos resíduos do açaí/juçara através } \\
\text { metodologias colaborativas ou pesquisas que incluam essas ferramentas para } \\
\text { aproveitamento de bioresíduos. }\end{array}$ \\
\hline & Critérios de exclusão & $\begin{array}{l}\text { Pesquisas duplicadas, pesquisas de acesso restrito, artigos que tratem do uso } \\
\text { da polpa ou das suas propriedades nutricionais do açaí ou pesquisas que } \\
\text { abordem o aproveitamento dos resíduos de açaí através de métodos } \\
\text { experimentais em laboratório. Tais pesquisas não deveriam estar relacionadas } \\
\text { à área da saúde, alimentação, formulação de substratos ou à testes químicos. }\end{array}$ \\
\hline
\end{tabular}

Quadro 1: Protocolo da RSL

Fonte: autora

Para a realização das buscas nas bases de dados foram escolhidas as seguintes palavras-chave: juçara, açaí, caroço, casca, semente, resíduos, colaboração, práticas colaborativas, design difuso, codesign, collaboration, collaborative practices, waste, seed. $\mathrm{O}$ 
quadro 2 apresenta as associações realizadas entre as palavras-chave, sendo que nas associações de \#1 a \#31 foram inseridas na base CAPES e nas de \#32 a \#45 foram inseridas na Base WEB OF SCIENCE.

\subsection{Conjunto de considerações iniciais}

A primeira etapa foi realizada entre o dia 13 de novembro a 10 de dezembro de 2019 e consistiu na busca e identificação dos artigos selecionados da seguinte maneira:

1) Base CAPES: foram identificadas 338 (trezentos e trinta e oito) pesquisas em português e 313 (trezentos e treze) pesquisas em inglês na busca avançada, com a inserção das associações de palavras-chave selecionando "QUALQUER”, “CONTÉM” ou "É EXATO”.

3) Base WEB OF SCIENCE - inglês: Foram identificados 180 (cento e oitenta) estudos na pesquisa básica com a inserção das associações de palavras-chave selecionando o campo “TÓPICO”.

A inserção de palavras-chave totalizou 831 pesquisas encontradas. Torna-se importante destacar que, conforme pode ser visualizado no Quadro 2, algumas buscas apresentaram mais de 200 (duzentas) pesquisas encontradas assinaladas com (*). Percebeu-se que esses resultados estavam relacionados à associação de palavras muito abrangente e por isso não foram considerados e nem contabilizadas para esta RSL.

Após a identificação geral de todos os estudos, foi realizada uma leitura rápida com base nos critérios para análise do material, conforme descrito no Quadro 1. Na sequência, eram descartadas as pesquisas que não se ajustavam aos critérios de inclusão do protocolo. $\mathrm{O}$ Quadro 2 abaixo apresenta a quantidade de pesquisas identificadas e selecionadas em cada associação.

\begin{tabular}{|llll|}
\hline Busca & Associação de palavras-chave & Identificados & Selecionados \\
\hline \#1 & Juçara AND semente & Base CAPES & \\
\hline$\# 2$ & Juçara AND casca & 10 & 0 \\
\hline$\# 3$ & Juçara AND caroço & 2 & 0 \\
\hline$\# 4$ & Açaí AND semente & 62 & 2 \\
\hline$\# 5$ & Açaí AND casca & 72 & 2 \\
\hline$\# 6$ & Açaí AND caroço & 28 & 2 \\
\hline
\end{tabular}


RELACult - Revista Latino-Americana de Estudos em Cultura e Sociedade

Revista Latinoamericana de Estudios en Cultura y Sociedad | Latin American Journal of Studies in Culture and Society V. 07, ed. especial, mar., 2021, artigo n ${ }^{\circ} 1996$ | claec.org/relacult | e-ISSN: 2525-7870

\begin{tabular}{|c|c|c|c|}
\hline$\# 7$ & Juçara AND colaboração & 5 & 0 \\
\hline$\# 8$ & Açaí AND colaboração & 11 & 0 \\
\hline \#9 & Açaí AND práticas colaborativas & 0 & 0 \\
\hline$\# 10$ & Juçara AND práticas colaborativas & 1 & 0 \\
\hline \#11 & Caroço AND práticas colaborativas & 0 & 0 \\
\hline$\# 12$ & Casca AND práticas colaborativas & 0 & 0 \\
\hline \#13 & Semente AND “práticas colaborativas” (é exato) & 1 & 0 \\
\hline$\# 14$ & Resíduos AND “práticas colaborativas” (é exato) & 8 & 0 \\
\hline$\# 15$ & Semente AND colaboração & 78 & 3 \\
\hline \#16 & Casca AND colaboração & 48 & 1 \\
\hline$\# 17$ & Juçara AND “co-design” (é exato) & 1 & 0 \\
\hline \#18 & Açaí AND “co-design” (é exato) & 2 & 0 \\
\hline \#19 & Caroço AND “co-design” (é exato) & 0 & 0 \\
\hline \#20 & Semente AND “co-design” (é exato) & 1 & 0 \\
\hline \#21 & Casca AND “co-design” (é exato) & 0 & 0 \\
\hline \#22 & Resíduos AND “co-design” (é exato) & 4 & 1 \\
\hline \#23 & Juçara AND collaboration & 11 & 1 \\
\hline \#24 & Açaí AND collaboration (últimos 10 anos) & 194 & 2 \\
\hline \#25 & Seed AND collaboration & $27 \mathrm{mil}^{*}$ & - \\
\hline \#26 & Waste AND collaboration & $56 \mathrm{mil}^{*}$ & - \\
\hline \#27 & Açaí AND “collaborative practices” (é exato) & 0 & 0 \\
\hline \#28 & Juçara AND “collaborative practices” (é exato) & 0 & 0 \\
\hline \#29 & Waste AND “collaborative practices" (é exato, últimos 2 anos) & 108 & 2 \\
\hline \#30 & Juçara waste AND “collaborative practices” (é exato) & 0 & 0 \\
\hline \#31 & Açaí waste AND collaborative practices (é exato) & 0 & 0 \\
\hline \multicolumn{4}{|c|}{ Base WEB OF SCIENCE } \\
\hline \#32 & Juçara AND seed & 5 & 0 \\
\hline
\end{tabular}


RELACult - Revista Latino-Americana de Estudos em Cultura e Sociedade

\begin{tabular}{|c|c|c|c|}
\hline \#33 & Juçara AND waste & 1 & 0 \\
\hline$\# 34$ & Açai AND seed & 47 & 0 \\
\hline \#35 & Açai AND waste & 24 & 0 \\
\hline \#36 & Juçara AND “co-design” (é exato) & 0 & 0 \\
\hline \#37 & Açaí AND “co-design” (é exato) & 0 & 0 \\
\hline \#38 & Juçara AND collaboration & 0 & 0 \\
\hline \#39 & Waste AND collaboration & $653^{*}$ & - \\
\hline \#40 & Açaí AND collaboration & 0 & 0 \\
\hline \#41 & Seed AND collaboration & $221 *$ & - \\
\hline \#42 & Seed AND collaborative practices & 28 & 0 \\
\hline \#43 & Waste AND collaborative practices & 75 & 0 \\
\hline \#44 & Juçara waste AND collaborative practices & 0 & 0 \\
\hline \#45 & Açaí waste AND collaborative practices & 0 & 0 \\
\hline Total & & 831 & 16 \\
\hline
\end{tabular}

Quadro 2: Artigos identificados e selecionados

Fonte: autora

Na base CAPES, várias pesquisas foram identificadas ao utilizar a associação entre as palavras "açaí" ou "juçara” e "caroço", "casca” e "resíduos”. Assim como na base WEB OF SCIENCE, ao utilizar as associações entre "açaí" ou "juçara" e "seed" e "waste". Isto porque essas são as palavras que formam a base para o presente artigo, ou seja, os resultados encontrados deveriam obrigatoriamente abrangê-las para que fossem identificados tendo em vista que são a matéria prima das pesquisas analisadas. Por outro lado, a maior parte das pesquisas encontradas estava relacionada a estudos experimentais para adubo, medicamentos e alimentos feitos a partir do caroço do açaí ou de sua polpa.

Considerando as pesquisas excluídas, grande parte delas estava relacionada às propriedades nutricionais da polpa da juçara e, mais frequentemente, do açaí. Enquanto que na base WEB OF SCIENCE, a maior parte das pesquisas excluídas estava relacionada ao estudo das propriedades medicinais presentes no fruto, incluindo polpa e caroço, para tratamento de doenças como o câncer. Estes e outros estudos não ofereciam resposta à pergunta de pesquisa e por isso não foram selecionados para a etapa posterior. 
Em ambas as bases, nas associações com as palavras "colaboração", "práticas colaborativas", "collaboration", "collaborative practices" e "co-design" as pesquisas encontradas não estavam relacionadas diretamente às práticas colaborativas realizadas entre a comunidade produtora de açaí e pesquisadores acadêmicos. Mas envolviam outros atores e apontavam a importância da inserção dessas práticas na transmissão e troca de conhecimentos tácitos e técnicos. Sendo assim, essas pesquisas foram selecionadas para uma análise mais aprofundada a fim de verificar se as informações nelas apresentadas poderiam ser utilizadas.

Base CAPES

651 identificados

16 selecionados

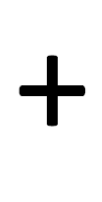

Somatório

831 identificados

17 selecionados

Figura 1: somatório de artigos identificados e selecionados

Fonte: autora

Na base CAPES, foram identificados 651 (seiscentos e cinquenta e um) estudos, sendo 16 (dezesseis) selecionados, enquanto que na base WEB OF SCIENCE foram identificados 180 (cento e oitenta) estudos, com apenas 1 (um) selecionado. Desse modo, como pode ser observado na figura 1 , foram selecionadas 17 (dezessete) pesquisas para uma análise mais criteriosa.

\subsection{Conjunto de considerações finais}

A última etapa da seleção foi iniciada por uma leitura criteriosa para identificar os estudos que mais se aproximassem da questão de pesquisa, tendo em vista que nenhum dos artigos encontrados estava diretamente relacionado a ela. Em sequência, foram analisados: o resumo, a introdução e a conclusão de cada artigo. Em alguns casos, foi necessário verificar a metodologia da pesquisa. Além disso, foram realizadas buscas utilizando a ferramenta de [“control" + "F"] pelas palavras-chave dentro do próprio texto do artigo. Esta ferramenta proporcionou encontrar informações importantes sobre o tema, as quais não estivessem presentes nos tópicos determinados anteriormente.

A leitura de um tópico evoluía para outro à medida que o texto apresentasse informações importantes relacionadas à questão da pesquisa. Ao final, os artigos selecionados foram subdivididos em dois grupos: incluídos e não incluídos. Na base CAPES, das 16 (dezesseis) pesquisas selecionadas apenas 2 (duas) foram incluídas. Enquanto que na base WEB OF SCIENCE, a única pesquisa selecionada foi descartada, pois estava relacionada à área da saúde. As pesquisas excluídas não atenderam aos critérios de inclusão descritos no 
item "d" do Quadro 1. E também não se adequavam perfeitamente aos critérios de exclusão que constam no item "e".

Ao verificar as pesquisas encontradas em busca de aporte teórico que permitisse a compreensão das práticas colaborativas que envolvessem a comunidade acadêmica e a comunidade local, detentora do saber tradicional, percebemos que existem poucos trabalhos que tratam sobre esse tema. Em geral, os estudos privilegiam a colaboração empresarial ou entre as diferentes áreas de conhecimento presentes numa universidade. Desse modo, foi imprescindível, incluir estudos que estivessem relacionados ao tema, ainda que não tratassem diretamente sobre ele. Essa busca teve o propósito de se apropria do que já vem sendo pesquisado no que se refere à valorização do conhecimento tácito de comunidades locais de modo a ampliar o entendimento de como essas relações podem ser efetivadas.

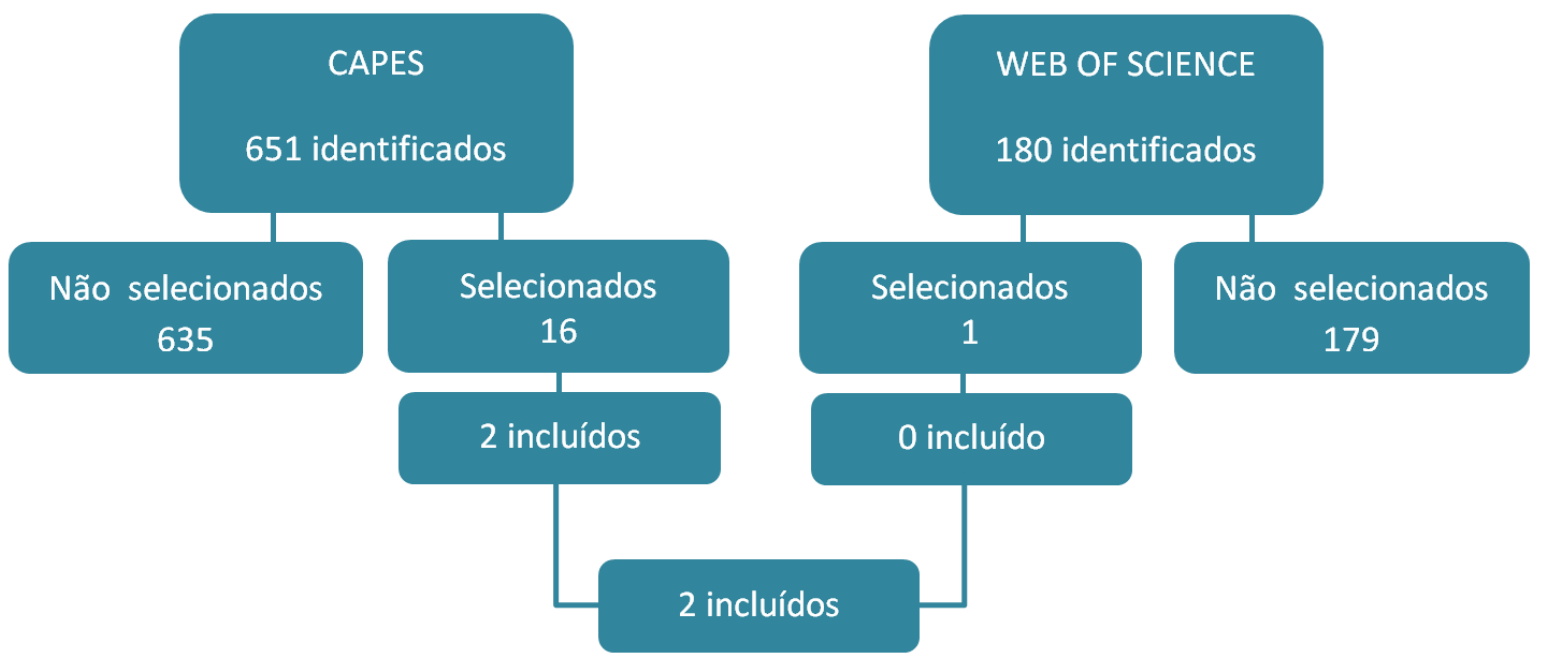

Figura 2: artigos incluídos nas Bases CAPES e WEB OF SCIENSE

Fonte: autora

Desta maneira, ao realizar a somatória do conjunto de consideração final das bases utilizadas, isto é, de todos os artigos incluídos, chegamos ao resultado de 2 (três) artigos em língua portuguesa na base CAPES.

\subsection{Análise descritiva}

A metodologia da Revisão Sistemática de Literatura proporcionou a identificação de 831 (oitocentos e trinta e uma) pesquisas, com a seleção criteriosa de 17 (dezessete) artigos para a contribuição teórica nesta pesquisa. Após leitura mais detalhada, foram incluídos os 2 (dois) artigos descritos no Quadro 3, abaixo: 
RELACult - Revista Latino-Americana de Estudos em Cultura e Sociedade

\begin{tabular}{|lllll|}
\hline $\mathbf{N}^{\mathbf{0}}$ & Título & Autor & Ano & Palavras-chave \\
\hline$\# 1$ & $\begin{array}{l}\text { Apropriação social da ciência e da } \\
\text { tecnologia: contribuições para uma } \\
\text { agenda. }\end{array}$ & $\begin{array}{l}\text { Maria Cristina Piumbato Hayashi } \\
\text { Innocentini; Cidoval Morais de Sousa; } \\
\text { Danilo Rothberg. }\end{array}$ & 2011 & $\begin{array}{l}\text { Semente (AND) } \\
\text { colaboração }\end{array}$ \\
\hline$\# 2$ & $\begin{array}{l}\text { Entre a etnoecologia e a silvicultura: o } \\
\text { papel de informantes locais e cientistas } \\
\text { na pesquisa florestal. }\end{array}$ & $\begin{array}{l}\text { Rafael Ricardo Vasconcelos da Silva; } \\
\text { Luiz Carlos Marangon; Ángelo } \\
\text { Giuseppe Chaves Alves. }\end{array}$ & 2011 & $\begin{array}{l}\text { Casca (AND) } \\
\text { colaboração }\end{array}$ \\
\hline
\end{tabular}

Quadro 3: Artigos incluídos

Fonte: autora

O estudo \#1 apresenta uma importante reflexão sobre a proteção de propriedades intelectuais de saberes tradicionais. A pesquisa aponta para o atual contexto em que se apresenta a dificuldade de controle e legislação sobre a apropriação de costumes tradicionais de comunidades locais. Por se tratar de um assunto relacionado ao tema em questão, é importante compreender como os saberes tradicionais têm sido abordados no âmbito nacional e internacional. A pesquisa demonstra que em países como o Peru, as comunidades encontram-se céticas em relação à efetividade das politicas de proteção de conhecimentos coletivos, ou seja, existe uma crise de confiança institucional entre comunidade e Estado. Mesmo com a existência de um banco de dados nacional para registro desses conhecimentos, existe uma desconfiança da apropriação indevida por terceiros. Países como Índia e China estimulam veemente o registro de conhecimentos tradicionais. Japão, Reino Unido e Alemanha adotam um sistema de que uma informação só anula a novidade de uma invenção se reproduzir o mesmo caminho e chegar ao mesmo resultado, caso contrário, atribuem legitimidade.

Sobre o tema, ainda não existem consensos, mas o debate é importante até que se encontrem as melhores soluções. $\mathrm{O}$ estudo traz como reflexão para a pesquisa em epigrafe a importância de garantir às comunidades que colaboram com pesquisas acadêmicas, o direito de ter seus conhecimentos resguardados. Para além da possiblidade de salvaguardar conhecimentos tradicionais de uma determinada comunidade, vale refletir também sobre a importância de proporcionar as condições necessárias para que eles se manifestem. Evitando com isso a imposição de soluções acadêmicas que não venham a ser absorvidos pela comunidade por não condizerem com sua cultura e suas tradições.

O artigo \#2 é desenvolvido com base nos trabalhos com foco no manejo, no uso e no conhecimento de recursos florestais por populações locais. Ele objetiva esclarecer a relação entre etnoecologia e silvicultura, considerando pesquisas em ambientes florestais e a relação da comunidade acadêmica, pesquisadores e engenheiros, com a comunidade local, os detentores dos conhecimentos tradicionais. O artigo apresenta os termos etnossilvicultura, 
etnoecologia e silvicultura sob a perspectiva de diversos autores, enfatizando que os desdobramentos da etnoecologia, como etnobotânica, etnossilvicultura e similares são na verdade cruzamento de saberes.

Silva, Marangon e Alves (2011) apresentam ainda a importância do papel do "mateiro", uma espécie de informante das plantas locais, o qual possui conhecimentos que podem ir além daqueles já descobertos cientificamente. Neste sentido, a pesquisa acadêmica pode ser enriquecida com a contribuição de pessoas de uma determinada comunidade considerando seu grau de ligação com a natureza e seus conhecimentos sobre o território. Além disso, as pesquisas etnoecologicas podem contribuir para aprofundar a compreensão dos usos e conhecimentos da floresta por meio de articulações e comparações entre os saberes locais e científicos. Explica ainda que algumas comunidades indígenas tem o costume de tratar a terra para o cultivo de plantas e que esse manejo sistémico não é uma criação moderna. Tal estudo (\#2) aponta para a importância de se considerar o conhecimento tradicional nas pesquisas cientificas e no quanto essa contribuição pode levar a novas descobertas.

\section{Conclusões}

O açaí possui diversas propriedades nutricionais e é amplamente consumido em todo estado brasileiro. Nativo da região amazônica tem como maiores produtores o estado do Pará, Amazonas e Maranhão. Considerando que 73,46\% do peso do fruto correspondem ao caroço, grande parte do fruto é descartada e chega a gerar mais de 161 mil toneladas de resíduos por ano. Sendo assim, na busca por soluções que venham a aproveitar esses resíduos na composição de novos materiais, as pesquisas encontradas apresentam uma forte ênfase nos ensaios laboratoriais, excluindo a comunidade detentora do saber tácito do processo de experimentação.

A Revisão Sistemática de Literatura - RSL adotada como ferramenta metodológica foi utilizada para identificar os estudos que envolvessem os resíduos do açaí na composição de novos materiais a partir de práticas colaborativas no processo de pesquisa. No entanto, nenhum estudo sobre o assunto foi encontrado. Dentre os 831 (oitocentos e trinta e um) estudos identificados na RSL, apenas 17 (dezessete) foram selecionados para uma leitura mais detalhada. Em seguida verificou-se que grande parte das pesquisas não atendia aos critérios estabelecidos na RSL, resultando em apenas 2 (dois) artigos incluídos, os quais faziam menção sobre a importância de se considerar e proteger os conhecimentos subjetivos de uma 
determinada comunidade.

Nos dois estudos percebeu-se que os saberes e os costumes tradicionais podem contribuir consideravelmente com os resultados das pesquisas a ser desenvolvida junto à comunidade. Enquanto a pesquisa \#1 reforça a importância de proteger esses saberes através de registros e patentes, a pesquisa \#2 aponta para a importância em considerar o conhecimento tácito das comunidades nativas, os quais devem ser empregados e comparados com os conhecimentos científicos já descobertos.

A presente artigo evidencia a ausência de pesquisas que considerem o conhecimento tácito no desenvolvimento de novos materiais no campo do design. Nenhuma das pesquisas relacionadas ao açaí e aos seus resíduos apresentou metodologias que incluíssem o conhecimento das comunidades tradicionais nos processos de experimentação. Por outro lado, fica evidente a necessidade de que esses atores sejam inseridos no processo de pesquisa através de práticas colaborativas, possibilitando o enriquecimento dos estudos acadêmicos para que venham a resultar em um aproveitamento mais sustentável dos recursos naturais através de novas possibilidades econômicas.

\section{Referências}

GALVÃO, Taís Freire; PEREIRA, Mauricio Gomes. Revisões sistemáticas da literatura: passos para sua elaboração. Epidemiologia e Serviços de Saúde, [s.1.], v. 23, n. 1, p.183-184, mar. 2014. Instituto Evandro Chagas. http://dx.doi.org/10.5123/s1679-49742014000100018. Disponível em: $\quad$ http://scielo.iec.gov.br/scielo.php?script=sci arttext\&pid=S167949742014000100018. Acesso em: 13 dez. 2019.

IBGE. INSTITUTO BRASILEIRO DE GEOGRAFIA E ESTATÍSTICA (Brasil). Sistema IBGE de Recuperação Automática. Tabela 289 - Quantidade produzida e valor da produção na extração vegetal, por tipo de produto extrativo. 2019. Disponível em: https://sidra.ibge.gov.br/tabela/289. Acesso em: 08 nov. 2019.

INGOLD, Tim. Estar vivo: ensaios sobre movimento, conhecimento e descrição. Petrópolis, RJ : Vozes, 2015.

JBIEBNM (Comp.). An Introduction to Systematic Reviews. Changing Practice: Evidence Based Practice Information Sheets fo, Adelaide, Sup. 1, p.1-6, 2004. Disponível em: https://www.semanticscholar.org/paper/Best-Practice-Evidence-Based-Practice-InformationBest-Vitales/b4f2a4b3747e196d86c0f2f53ee05d0c7c5d8db5. Acesso em: 13 dez. 2019.

MANZINI, Ezio. Design: Quando todos fazem Design - uma introdução ao design para a inovação social. Tradução de Luzia Araujo. São Leopoldo: Ed. UNISINOS, 2017. 254p. 
MARÇAL, Tiago de Souza; FERREIRA, Adésio; OLIVEIRA, Wagner Bastos dos Santos; GUILHEN, José Henrique Soler; FERREIRA, Marcia Flores da Silva. CORRELAÇÕES GENÉTICAS E ANÁLISE DE TRILHA PARA CARACTERES DE FRUTO DA PALMEIRA JUÇARA. Revista Brasileira de Fruticultura, [S.L.], v. 37, n. 3, p. 692-698, set. 2015. FapUNIFESP (SciELO). http://dx.doi.org/10.1590/0100-2945-163/14. Disponível em: https://www.scielo.br/scielo.php?script=sci_arttext\&pid=S0100$29452015000300692 \& \operatorname{lng}=$ pt\&tlng=pt. Acesso em: 20 dez. 2019.

MARTINS, Maria Alice; MATTOSO, Luiz Henrique Capparelli; PESSOA, José Dalton Cruz. Comportamento térmico e caracterização morfológica das fibras de mesocarpo e caroço do açaí (Euterpe oleracea Mart.). Revista Brasileira de Fruticultura, [s.1.], v. 31, n. 4, p.11501157, dez. 2009. FapUNIFESP (SciELO). Disponível em: https://www.scielo.br/scielo.php?script=sci_arttext\&pid=S0100-

$29452009000400032 \& \operatorname{lng}=$ pt\&tlng=pt. Acesso em: 20 dez. 2019.

MESQUITA, Antonio de Lima; BARRERO, Núbia Garzon; FIORELLI, Juliano; CHRISTOFORO, André Luís; FARIA, Lenio José Guerreiro de; LAHR, Francisco Antonio Rocco. Eco-particleboard manufactured from chemically treated fibrous vascular tissue of acai ( Euterpe oleracea Mart. ) Fruit: a new alternative for the particleboard industry with its potential application in civil construction and furniture. Industrial Crops And Products, [S.L.], v. 112, p. 644-651, fev. 2018. Elsevier BV. http://dx.doi.org/10.1016/j.indcrop.2017.12.074. Disponível em: sciencedirect.com/science/article/abs/pii/S0926669017309044?via\%3Dihub. Acesso em: 20 dez. 2019.

OLIVEIRA, Maria do Socorro Padilha de; CARVALHO, José Edmar Urano de; NASCIMENTO, Walnice Maria Oliveira do; MÜLLER, Carlos Hans. Cultivo do Açaizeiro para Produção de Frutos. 2002. Ministério da Agricultura, Pecuária e Abastecimento. Circular Técnica $26 . \quad$ Disponível em: https://www.infoteca.cnptia.embrapa.br/infoteca/handle/doc/405768. Acesso em: 30 nov. 2019.

OLIVEIRA, Maria do Socorro Padilha de; FARIAS NETO, João Tomé de. ÁRVORE DO CONHECIMENTO: Açaí. Agência Embrapa de Informação e Tecnologia - ageitec. Disponível

em: https://www.agencia.cnptia.embrapa.br/gestor/acai/arvore/CONT000gbfan1e702wx5ok07shn q97wntcgb.html. Acesso em: 20 dez. 2019.

REPÓRTER Mirante destaca a cultura em torno na juçara no Maranhão. G1 Maranhão, São Luís, 26 out. 2019. Disponível em:

https://redeglobo.globo.com/ma/tvmirante/noticia/reporter-mirante-destaca-a-cultura-emtorno-na-jucara-no-maranhao.ghtml. Acesso em 20 dez. 2019.

RIGOLIN, CCD. Biotecnologia e sistemas de conhecimento: propostas de regulação. In HAYASHI, MCPI., SOUSA, CM., ROTHBERG, D., orgs. Apropriação social da ciência e da tecnologia: contribuições para uma agenda [online]. Campina Grande: EDUEPB, 2011. pp. 41-106. ISBN 978-85- 7879-187-2. Available from SciELO Books. Disponível em: http://books.scielo.org/id/j76hp/pdf/hayashi-9788578791872-03.pdf. Acesso em: $20 \mathrm{dez}$. 2019. 
SANCHES, Leonardo; ABDALLA, José Gustavo Francis; ALBERTO, Klaus Chaves. As pesquisas sobre sistemas generativos: uma revisão sistemática de literatura. Parc Pesquisa em Arquitetura e Construção, [s.1.], v. 9, n. 2, p.133-151, 29 jun. 2018. Universidade Estadual de Campinas. http://dx.doi.org/10.20396/parc.v9i2.8650200. Disponível em: https://periodicos.sbu.unicamp.br/ojs/index.php/parc/article/view/8650200. Acesso em: 13 dez. 2019.

SANDERS, E. B. N. From user-centred to participatory design approaches. In: J. Frascara (Ed.). Design and the social sciences: Making connections, London, 2002. n.p.

http://maketools.com/articles-papers/FromUsercenteredtoParticipatory_Sanders_\%2002.pdf.

Acesso em: 25 set. 2019.

SILVA, Rafael Ricardo Vasconcelos da; MARANGON, Luiz Carlos; ALVES, Ángelo Giuseppe Chaves. Entre a etnoecologia e a silvicultura: o papel de informantes locais e cientistas na pesquisa florestal. Interciencia: Asociación Interciencia, Caracas, Venezuela, v. 36, n. 7, p.485-492, 7 jun. 2011. Disponível em: https://www.redalyc.org/articulo.oa?id=33919424003. Acesso em: 20 dez. 2019.

SPINUZZI, Clay. The methodology of participatory design. Applied Research. Volume 52, Number 2, May 2005, p.163 a 174. Disponível em:

https://repositories.lib.utexas.edu/bitstream/handle/2152/28277/SpinuzziTheMethodologyOfP articipatoryDesign.pdf?sequence $=2$ 